\title{
La investigación jurídica en el proceso de enseñanza-aprendiraje, un desafio en la carrera de Derecho, de la UNAN-Managua
}

\author{
Lic. Leonardo D. González Estrada \\ UNAN-MANAGUA \\ leochorotegano@yahoo.com \\ leochorotegaunan@gmail.com
}

DOI: https://doi.org/10.5377/torreon.v7i18.7717

Palabras claves: Investigación jurídica, enseñanza aprendizaje, modelo educativo UNAN-Managua.

\section{RESUMEN}

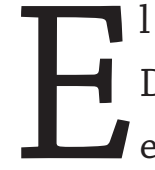

1 proceso enseñanza-aprendizaje de la investigación jurídica, en la carrera de Derecho de UNAN-Managua, desde su entrada en vigencia del actual modelo educativo 2011 en el 2013, es un desafío, porque implica una actualización en todos los ámbitos de formación, para ambos protagonistas: estudiantes y docentes. La presente evaluación y crítica dentro de un plazo prudencial de siete semestres, se consideró para conocer el contexto de la investigación jurídica, los avances, sus debilidades y fortalezas, como el comportamiento de conformidad al modelo educativo en ciernes. Aquí se alcanza a observar que, la pedagogía jurídica y la investigación científica del derecho son contenidos curriculares tradicionales, también teóricos, técnicos y metodológicos que deben ser acercados y adecuados a los procesos epistémicos jurídicos desarrollados en Latinoamérica.

\section{INTRODUCCIÓN}

Esta investigación parte de la línea de investigación número 3, para desarrollo institucional de la Universidad Nacional Autónoma de Nicaragua de Managua (UNAN-Managua), que prescribe como la "Validación de la aplicación de estrategias de enseñanza- aprendizaje 
en la UNAN-Managua". De tal manera que, realiza una evaluación en el contexto y procesos de formación de la investigación jurídica, a partir de la experiencia de docentes y estudiantes en la enseñanza-aprendizaje de aquellas asignaturas que tienen como propósito desarrollar la consciencia científica, desde la investigación en la carrera de derecho, como de la realidad curricular y planes de estudio sobre la investigación en la carrera.

Por esto se indica que, la enseñanza-aprendizaje del Derecho en Nicaragua, su pedagogía y metodología tienen su propia característica, pero la perspectiva de formación con bases en la investigación científica es todavía un desafío, como la implementación del modelo mismo que ha asumido la UNAN-Managua a partir del 2011, para la formación general de la Licenciatura en Derecho, el cual debe estar sustentado en supuestos, fundamentos, principios, didácticas, técnicas, pedagogías y metodologías propias para su concreción.

Lo anterior es el contexto e indicios tangibles de la transición en la enseñanza en la Educación Superior de Nicaragua. Se debe decir, que no existen antecedentes especializados sobre la Metodología de la investigación en la Ciencia del Derecho en Nicaragua, tampoco estrategias didácticas de enseñanza o aprendizajes del derecho basadas en la investigación jurídica. Sus métodos ni pedagogías son producto de los espíritus de los juristas nacionales, no de las estrategias de las escuelas de derecho nacionales o de la UNAN-Managua.

Para la fundamentación teórica y análisis hermenéutico, desde la práctica pedagógica para la investigación jurídica y sus metodologías, como antecedentes, señalan autores tales como Witker (1996), Fix-Zamudio (2007) y de León Armenta (2005) -entre otros más-, que esta se encuentra ampliamente desarrollados en Nuestra América con criterios que son la base para la propuesta en la nueva formación del Derecho e investigación jurídica. Cabe destacar, que Chile también tiene tradición en teorías, como ahora Argentina, Colombia, Perú y Venezuela, estas últimas con mucha heterogeneidad, en tanto, lo demanda la propia Ciencia del Derecho.

Sobre el contexto nicaragüense, la investigación jurídica, realizada por juristas reconocidos por toda la sociedad nicaragüense, en lo general, no se expone fuera del paradigma exegético, normativista y positivista, pero podría destacarse el aporte de un jurista, que podría ser el punto de partida en la investigación de la ciencia del Derecho, juristas como Solorzado (1999) y Escobar Fornos (2000) (a partir de los noventas y la actualidad), que implementan la metodología jurisprudencial, la doctrina enciclopédica y derecho comparado, pero a la vez positivista; el primero, realizó sus aportes jurídicos al Derecho Mercantil nicaragüense, haciendo una propuestas de glosas al código de Comercio, es decir, centrada su investigación al espíritu de la norma y regulación, con una técnica clásica del derecho, la glosa de la norma. Escobar también, pero este al Derecho civil o teoría contractual, también a las leyes del Registro Público, todo esto centrado a la interpretación de la norma. Montiel Argüello (1971), aporta significativamente a la 
construcción del derecho en su obra “Jurisprudencia Civil Nicaragüense”, también está Huembre y Huembes (1971) y su obra "Nuevo Diccionario de Jurisprudencia Nicaragüense”, ambos son referentes de los métodos y técnicas en el derecho en su aplicación jurisdiccional.

La implicancia y vinculación de la enseñanza-aprendizaje e investigación científica del Derecho, desde estrategias curriculares, didácticas jurídicas, podría significar para la sociedad nicaragüense, el nivel cualitativo -léase institucionalidad, legalidad y legitimidad en cada acto o hecho social- en tanto la existencia de una praxis sociológica del derecho, modulada por y desde la las técnicas de la enseñanza y la metodología en la investigación jurídica, como eje estratégico del modelo enseñanza-aprendizaje del Derecho, en la UNAN-Managua, se convierte en un contenido estratégico para llevarlo a cabo.

Lo anterior es una necesidad e imperativo de realizar una investigación que sistematice, estructure y proponga la valoración inicial sobre la enseñanza-aprendizaje, que aporte en la validación al actual modelo de enseñanza, el que tiene por propósito, además, formar al estudiante de grado, a partir de didácticas metodológicas jurídico-sociales para la formación en investigación jurídica científica.

En esta investigación se consideran dos categorías principales, en la cual, una corresponde a uno de los ejes del modelo educativo de la institución; y la otra, el fundamento genérico del mismo modelo, la enseñanza-aprendizaje del derecho e investigación jurídica. Se puede decir, que esta investigación será exploratoria analítica, con un enfoque cualitativo, en tanto, las valoraciones de mayor peso, serán de juicio cualitativo de los protagonistas, o sea del docente y del estudiante que construyen conocimiento, que participan en el proceso enseñanza-aprendizaje en la estrategia de investigación del derecho, también desde la hermenéutica-constructiva de la teoría.

\section{MATERIALES Y MÉTODOS}

En cuanto al enfoque de la presente investigación, por el mayor énfasis en el uso de información cualitativa, esta investigación se realiza mediante la aplicación del Enfoque Filosófico Cualitativo de Investigación (Pedroza, 2017). En cuanto al nivel de compromiso de investigador, la investigación se adhiere al Paradigma Socio-constructivista, de acuerdo a la postura de Ferreiro (2004), puesto que se ocupa de cómo se adquiere el contenido de enseñanza, también cómo se transita de un estado de conocimiento inferior a otro orden superior, más aún, cómo se forman las categorías de pensamiento.

Se aplicó como técnica cualitativa de investigación, la entrevista en profundidad de tipo analítica, a cinco docentes que tienen tradición docente en la UNAN-Managua, además de las cátedras sustantivas del derecho, en aquellas relacionadas con la metodología de investigación 
científica para el derecho o que pedagógicamente tengan relación o compromiso con la investigación en la carrera, y una encuesta no paramétrica a cincuenta estudiantes de cuarto año de la carrera. Procurando, al decir de Álvarez-Gayou (2003), “obtener descripciones del mundo -jurídico en el caso concreto- del entrevistado respecto a la interpretación de los significados de los fenómenos descritos" (p.109).

\section{RESULTADOS Y DISCUSIÓN}

Para la descripción del proceso desde la investigación jurídica, el $78 \%$ define su enseñanzaaprendizaje en investigación jurídica como Teórico práctico. Juicio de valor dado por el estudiante, el cual tuvo dos opciones más, o sea, que pudieron elegir que el proceso en el que se desarrollan como abogados, siendo estudiantes, es Teórico o Científico. De tal manera que, este criterio es parte de la concepción generalizada desde la formación empírica del estudiante, donde por el mismo orden y diseño del currículo este oferta asignatura como Prácticas de profesionalización, especialización o de familiarización, por lo cual, el aspecto práctico, y en suma teórico-práctico obedece principalmente, a esa estrategia de relacionar al estudiante y algunos de sus contenidos con la lógica institucional del ente estatal o privado, al cual el estudiante vaya a realizar aquellas prácticas. No hay vinculación de esa estrategia con la investigación jurídica propiamente.

Por otro lado, y asumiendo el criterio de los protagonistas, si no fuera por las prácticas de profesionalización, práctica civil, práctica penal y notarial, estaríamos ante una formación puramente teórica, y desvinculada plenamente de la investigación científica del derecho. La carrera asume el ámbito empírico, aquel donde el estudiante desarrolla las habilidades de litigación y representación legal; dentro de este orden se puede decir, que se está ante "un modelo de antecedentes medievales, expresa Witker (1985), pues es una técnica de enseñanza autoritaria radicada en el profesor, que monopoliza la información, el conocimiento y el método, relegando a los estudiantes a un papel pasivo y receptivo".

Por su parte, los informantes claves, admiten que los estudiantes presentan interés por la investigación, es decir, superan las condiciones curriculares y de formación docente, hasta institucionales. Comportamiento estudiantil, asociado al modelo educativo del 2011, y los planes de estudio del 2013. Cuando estos expresan que el 72 \% de los estudiantes consideraría la investigación en su orden de prioridades académicas; en primer lugar, están llamando a que les formen en investigación jurídica, porque mientras el docente procura el camino más fácil o rápido, como primer criterio, evitar evaluar investigaciones, ya que implica un menor esfuerzo docente, los protagonistas principales del modelo educativo, la formación en investigación jurídica pues, será una materia pendiente, para el plan académico curricular de la carrera de derecho de UNAN-Managua. 
Al determinar los contenidos para una formación científico-jurídica, desde los roles implementados por los protagonistas del proceso de enseñanza-aprendizaje en la carrera de Derecho de UNAN-Managua, el informante clave menciona que se mejoró o amplió la propuesta de asignaturas para la formación y desarrollo de la investigación, sin poder establecer una concatenación sistémica entre asignaturas, tiempos y estrategias, cuya relación y sustento es la búsqueda del nuevo modelo educativo, que debe seguir supuestos pedagógicos socioconstructivistas, tal como propone Ander-Egg (1995), sobre las características principales: 1) Conocimientos previos; 2) Aprendizajes significativos; 3) El Estudiante protagonista; 4) Acompañamiento docente e institucional, para alcanzar los Aprendizajes construidos. Sin embargo, el socio-constructivismo de Ander Egg no intermedia en el proceso de formación, esto en virtud de lo que señalan los protagonistas, solo el $8 \%$, es decir, cuatro estudiantes, tuvieron un acompañamiento completo del $100 \%$ en las investigaciones.

Por ello y para esos propósitos, los profesores y estudiantes tienen que asumir papeles diferentes a los que tradicionalmente han desempeñado, recuperando para ellos mismos el derecho a la palabra y a la reflexión sobre su actuar. De tal manera que, los contenidos, para superar aquella enseñanza tradicional, el estudiante, protagonista, los asume desde la estrategia "aprender haciendo" en el proceso de enseñanza-aprendizaje, que busca la efectividad del modelo educativo, con una formación activa, científica y técnica de estudiante.

Si los estudiantes acreditan que su formación la define como teórico-práctico; además, confirman el 66 \% de los protagonistas, que tienen un acompañamiento docente en sus investigaciones. De esto se deduce que hay contenidos que hacen que los estudiantes investiguen, y que los docentes que imparten las asignaturas de investigación, además de acompañar, forman en investigación.

La valoración por parte de los informantes claves (docentes) y de los protagonistas (estudiantes), tomando como punto de partida la epistemología jurídica pedagógica y científica sobre el proceso de enseñanza-aprendizaje de la investigación jurídica en la carrera de Derecho, desde el enfoque del actual modelo educativo de 2011 y su malla curricular vigente desde el 2013, en la cual, la síntesis de Witker (2008), que dice que debe insistirse en la interdisciplinariedad como transdisciplinariedad, es decir, incorporar el pensamiento complejo para que logre un trabajo con médicos, con economistas, estudiar el derecho laboral como parte del derecho de empresa, convirtiéndose esto en la premisa principal para el presente contexto.

Aunado lo anterior a lo expresado por Ander Egg (1995), quien dice que el aprendizaje se nutre de la ayuda externa de parte de los educadores y de otros compañeros -por cuanto, todos los protagonistas del sistema educativo, son inmediatamente co-responsables del conocimiento 
aprendido/construido, lo que debería tener relación con el aporte, es sustancial, porque los estudiantes han realizado cincuenta y cuatro investigaciones, con las variaciones descritas.

Sin embargo, no hay relación con la propuesta latinoamericana y epistémica con los demás resultados y hallazgos expresados por los participantes, pues el 92 \% de los estudiantes no ha participado en Foros o Jornadas de Desarrollo Científico; además, si el 62 \% de estudiantes dice no haber adquirido habilidades de investigador, y un 78 \% de estudiantes, dice que la Carrera de Derecho, sí tiene las condiciones para formarlo como investigador en el Derecho, entonces, al haber una diferencia significativa entre las variables de categorías, se está ante condiciones subjetivas como institucionales óptimas para la investigación en la carrera de Derecho, a pesar de las contradicciones y articulación interna.

Y en tanto que, se adecue la investigación jurídica a una lógica más concreta y social, y según el aporte de Ragin (2007), a finalidad concretas del derecho como: 1) Identificar el comportamiento del sujeto o sectores sociales en relación a la ley en lo particular y general; 2) Evaluar la efectividad de la ley, doctrinas, valores y filosofía del derecho; 3) Hacer predicciones en lo atinente al proceso de formación de la ley, criterios estructurales y sociales integradas a la ley y medir la efectividad de la ley; 4) Desarrollar la hermenéutica y el socio constructivismo jurídico, sociología, filosofía y deontología del Derecho; 5) Explorar la heterogeneidad del Derecho desde lo preceptuado por el método científico; 6) Integrar a los sujetos sociales, jurídicos y políticos, como protagonistas en las investigaciones; y 7) Hacer progresar las teoría y tratados del Derecho.

En suma, la necesidad de que el docente abandone la actitud del monólogo y emprenda un diálogo con sus estudiantes, de manera que estos, participen en discusiones, redacten monografías, efectúen labor de seminario. Esta propuesta de Fix-Zamudio (2006), sí podrá ser parte de la estrategia en investigación de la carrera, una vez que también se considere la formación de los docentes en investigación social y en epistemología de las ciencias, constituyéndose en las bases necesarias, para el desarrollo del conocimiento socio jurídico. 


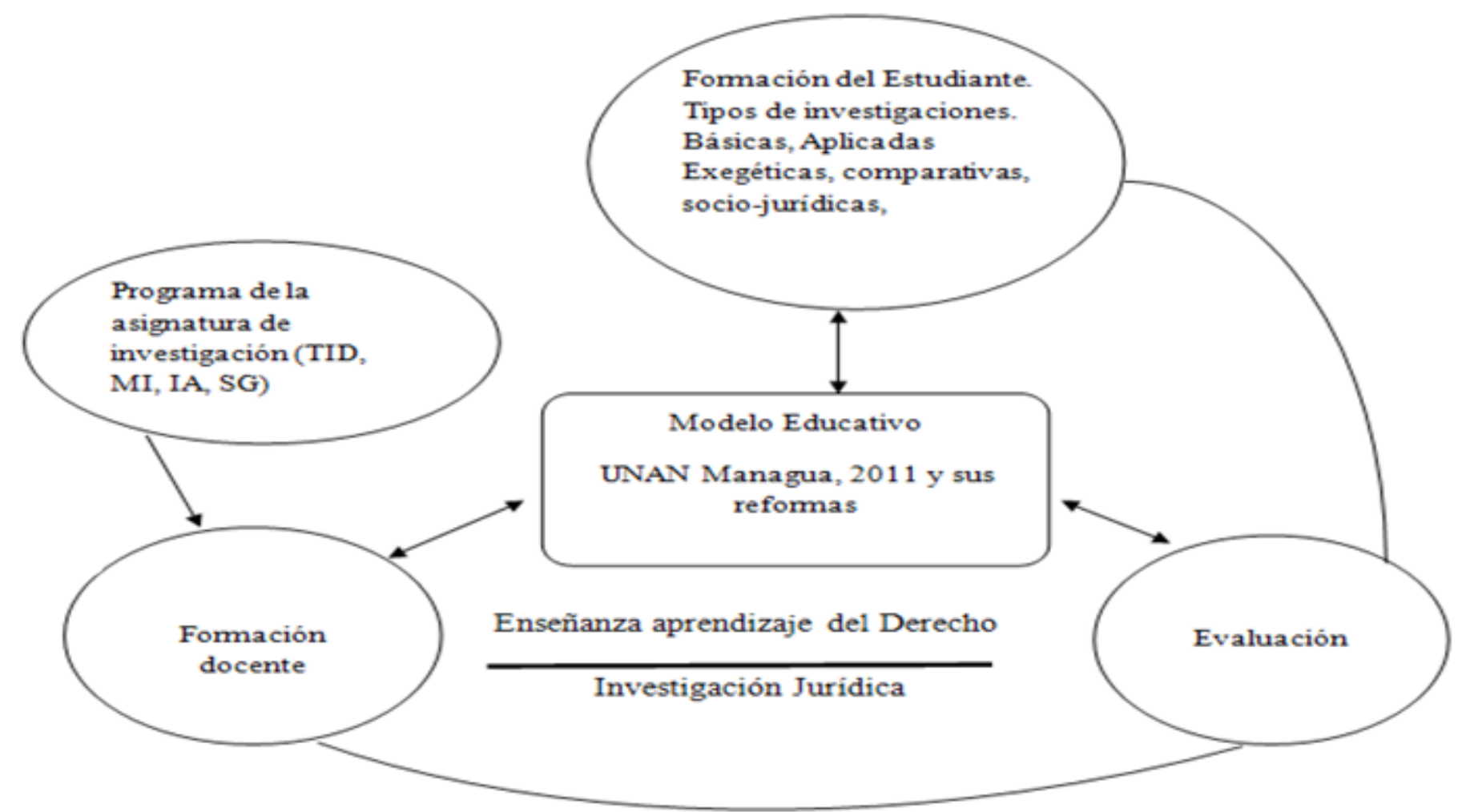

Figura 1

En este esquema se encuentran representadas aquellas categorías, que son las condiciones institucionales, curriculares y pedagógicas, como protagonistas del modelo educativo de la UNAN-Managua.

Se debe centrar en identificar la debilidad principal, en la cual la enseñanza-aprendizaje no contiene la investigación jurídica, es decir, subyace una disyuntiva entre los contenidos tradicionales, los contenidos del plan académica 2013 y lo que realmente demanda una formación jurídica, científica y la multidimensionalidad epistémica, que se ha avanzado sobremanera en otras naciones latinoamericanas.

Otro factor identificado es la formación docente y la evaluación en investigación jurídica el cual es un contenido pendiente para la carrera, lo que determina la dependencia y relación con los demás presupuestos del Modelo Educativo de la UNAN-Managua (2011).

De tal manera que, la formación del estudiante en investigación jurídica dependerá de la formación docente, de los contenidos curriculares, así como del tipo de evaluación propia, para que la relación entre formación del estudiante y acompañamiento docente sea significativo, constructivo y sobre todo, pedagógico como científico.

Sobre esta realidad, cabe resaltar que con el Modelo Educativo de 2011 y sus respectivos planes, también proponen a las unidades académicas nuevas metodologías para la evaluación 
en correspondencia con este. Sin embargo, las carreras más tradicionales, como la carrera de Derecho, presenta ciertas características, pues los docentes no tienen formación docente específica ni formación en investigación jurídica. Esto repercute en, cerrarse y centrarse en el paradigma positivista de la enseñanza, de tal manera que, cualquier dato o información y participación de protagonistas en investigaciones, todavía tendrá cierta resistencia.

\section{REFLEXIONES FINALES}

Se logra identificar que la pedagogía del derecho, en la escuela de Derecho de la UNANManagua parte de un proceso de enseñanza-aprendizaje de los contenidos de cada asignatura, a partir siempre de la tradición. Son casi inexistentes los hallazgos de contenidos teóricos, didácticos y metodológicos sobre pedagogía jurídica nicaragüense o epistemología jurídica para la investigación.

En la Carrera de Derecho, no se forma en el método de investigación científica con las bases metodológicas propias del Derecho, corriente iniciada y desarrollada en México. Existe un sesgo pedagógico institucional, hacia la formación positiva, normativa y axiológica del Derecho, lo que tiene por efecto, que el estudiante de Derecho pierde grandes oportunidades, para formarse científicamente.

Con relación al desarrollo y contenidos epistemológico de la ciencia del Derecho, no se está aprovechando la oportunidad que significa el actual Modelo Educativo de 2011 (modificado en 2015), para la formación desde la epistemología del derecho desarrollada en varios países Latinoamericanos, para lo cual, cabe la adecuación curricular en toda la estrategia de investigación en el derecho, desde el enfoque interdisciplinario y multidimensional.

\section{REFERENCIAS BIBLIOGRÁFICAS}

Álvarez-Gayou, J. L. (2003). Cómo hacer Ferreiro, R. (2004). Más allá de la teoría: El investigación cualitativa. Fundamentos $y$ metodología. México, D.F.: Paidos educador.

De León Armenta, L. P. (2005). Metodología del derecho. Editorial Porrúa. México.

Egg, E. A. (1995). La planificación educativa. Conceptos, métodos, estrategias y técnicas para educadores. Río de la Plata: Magisterio del Río de la Plata. Aprendizaje Cooperativo: El Constructivismo Social. El modelo educativo para la Generación N, Nova Southeastern University.

Fix Zamudio, H. (2007). Metodología, docencia $e$ investigación jurídicas. Editorial Porrua Argentina. Recuperado de: https://www. corteidh.or.cr/tablas/r32767.pdf

Huembre y Huembes. J. (1964). Nuevo diccionario dejurisprudencianicaragüense. $1^{\text {a }}$ edición. Imprenta Nacional Obligaciones. Hispamer. 
Montiel Arguello, A. (1971). Jurisprudencia Civil Nicaragüense. Tomo I. Impreso en los Talleres de la Imprenta Nacional, Managua, DN, Nicaragua.

Pedroza, P. M. (2017). Curso de Metodología de investigación. Farem-Carazo. UNANManagua.

Ragin, C. C. y de Setién Ravina, C. M. (2007). La construcción de la investigación social: introducción a los métodos y su diversidad. Siglo del Hombre Editores.

Solorzado, R. A. (1999). Glosas al Código de comercio de Nicaragua: Concordancia y jurisprudencia. Tercera edición. Hispamer.

Universidad Nacional Autónoma de Nicaragua de Managua. (2011). Modelo Educativo. Consejo Universitario. Editorial Universitaria. (Modificado en Octubre de 2015).
Witker, J. (2008). Hacia una investigación jurídica integrativa. Obtenido de Boletín Mexicano de Derecho Comparado. Disponible en: http://www.redalyc.org/ pdf/427/42712213.pdf

Witker, J. (2008). Metodología de la enseñanza del derecho. Porrúa.

Witker, J. (1996). Técnicas de Investigación Jurídica. Instituto de Investigaciones Jurídicas, Universidad Nacional Autónoma de México, Editorial.

Witker, J. (1985). Técnicas de la enseñanza del derecho. (4a edición). México: Editorial PAC. Obtenido de: http://archivos.juridicas. unam.mx/www/bjv/libros/3/1070/1.pdf 